\title{
Ueber die Einwirkung der Schwefélsăure auf fette Körper
} von Edmund Fremy. )

(Von dem Vertasser milgetheilt.)

Schon seit langer Zeit ist es bekinant, dafs die Schwefelsäure mit fetten Rörpern eine Art von Verseifung eingeht, und man hat selbst dem Prodncte dieser Einwirkung den Namen Schwefelsäure-Seife (savon sullyarique) gegeben.

Marquer erwähnt in seinem Dictionnaire de Chimie einer schwefalsauren Seife. Braconnot und Caventou beschäftigten slch cbenfalls mit der Behendlung fetter Rörper mit Schwefelsäure. Vorziiglich aber ist es Chevrcul, welchem wir bestimntere Angaben über diesen Gegenstand verdanken. Wir linden in sciner Abhandlung über die fetten Körper die Producte bcschrieben, welche die Schwefelsäure

oine grofse Zohl von Analysen wiederholl und immer ihnliche Resultate erhalten hatien. natmen wir an, ders das Stearin, betrachtet nls Stearo-Glycerlnsiture, ein Hyarat seyn und 2 Alone Wesser enthalten müsse, diesnlbe menge, wolehe die Greie Stoaringliupe enthalt. - Anmalen der Pharmacie Bd. XIX. S. 264 .

Wir goben diese Arbeit sur Verrollständigung der hursen No. tir, woh he wir in diesen Annalen Bd XIX. S. 296. milgetheilt habem. Mit einer seltenen Ausdauer hat Herr Fremy Licht in einige der verwickeltsten Verwaltris der Zerselzung enga nischer Körper gebracht, doch jetzt sehen wir dafs die Wir. kung der Säuren auf die felten Körper so gut wie unbckarint, war, und jedem muls es klar werden, dafs, wo man auch in der organischen Chemie angreifon mag, ubberall wird man wich. tige und erfolgreiche Enttlockungen zu erwarten haben, namentlich wean die strengste Gowissenhaftigkeit in der Erklärung der Ersrtieinmgen stets mit der Elementaranalyse gleiehen Schrilt halt.

D.I. 
in Berührung mit Oelen bitdet. Er fibrt die Bildung ron Tettsăuren, sowie eine Verbindung des Oels nit Sture an, welche er Sulfo-adipic-Säure nenint, und gedenkt exldlich der Höchst wahrscheinlichen Bildung ron Glycerin.

Die Untersuchungen von Che vreul über dtesen Gegenstand interatitzten wich wesentlich in der Erforschnng der Thatsachen. welche vorzulegen ich im Begrilfe hin, und nur indem ich densclben Weg einschlog, welchem er in seinen schönen Arbeiten über die Fettkörpor gefolgt ist, golang es mir. die oft zahlreichen Schwierigkeiten au ubberwinden, wolche dle Unfersuchung ron Körpern darbiotet, doren Studiam ith ru meinem Zwecho gemach hatte.

In dieser Abhandlung wer de ich nur von der trinwlithing der Schwefelsäure aut Oele sprection, and mic als zweite Arboit vorbehalten atich andere fette Körper denselben Versuchen zu unterwerfen. Doch mag jetzt schon erwähnt werden, dor's fast alle Fettkbrper, welche ich onf äbnliche Weise behandelt habe, sich nahezu wie die Oele verhielten. Ohgleich ich reruchiedene Oele der Einwirkang der Schwefelsüure unterworfen habe, so sind es doch vorzaglich das Olitenol und das sorise Mandelöl, welche mir zu dea nachfolgenden Versuchen dienten, und ich werde ein- und för allemal das Verhalten des Olivenöls als Beispiel annehmen.

Vorerst glaube ich noch auf die Zusammensetzung dieses Oels zurückkommen to mässen. um die Art der Einwirkung, nelche die Schtrefelsäure auf dasselbe ausübt, verstïndlicher zu geben.

Man weifs, dars dieses Oel einen flüssigen und einen festen Korper, das Olein und das Margarin, enthält. Die Versuche, welcho math über die Verseifung des Oels angestellt hat, und die ich mit aller Sorgfalt wiederholt habe, belebren uns, dafs aus dem Oele hierbei zwei Säuren, die Olein- und Margarinsiure, und aufserdem noch Glycerin entsteten, wonach sich 
dasselbe ols oleinsaures und margarinsauras Glycerin betrachten lielse. Wenn man nun das Oel mit concentrirter Schwefelsäure behandelt, so wird es in seine drei Bestandtheile zerlegt, indem dio Schwefelsäure sich mit den beiden Fottsüuren zu zwci neuen Säuren rerbindet, welche ich Sulfomargarinsäure und Sulfooleinsäure nennen werde. Das Gly. cerin verbindet sich ebenfalls mit Schwefelsäure, und bildet die Sullnglycerinsiure, welche Pelouze in der letzteren Zeit untersuebt hat.

Ich gehe nun zu den Einzelnheiten der Versuche über. Man bann ein bis zwei Pfunde Olivenöl in Behandlung nehmen, ohne dasselhe mit einer Kältemischung umgeben zu müseen, um eine Temperaturerhöhung za verhindern, welche die Entwichelung ron schwefliger Säure veranlassen würde. Ich habe gelunden, dafs man dem Oele nur sein balbes Gewicht concentrirter Schwefelsäure, jedoch in kleinen Mengen und in Absätzen ron einigen Minuten, hinzuzusetzen brancht, um bei Befolgung dieser Vorsichtsmaforegeln der Entwickelung auch der Lleinsten Spuren von schwefliger Säure vorzubeugen.

Wälırend dieses Verlahreas hat sich das Oel ein wenig gefärbe, und ist seiner Masse nach sehr dick geworden. Man muifs das Ganze nun mit seinem zweifachen Volumen Wasser behandeln, norauf die heiden Süuren, die Sulfomargariu. und Sulfooleinsüure, in Gestalt eines dichen Syrups auf der Oberfüche schwinmend erscheinen, während dic Sulfoglycerinsaure mit der überschüssigen Schwefelsäure in dem Wasser aufgelöst bleibt. Man liann die Anwesenheit dicser Säure lejcht nachweisen, ஷ̀̀eno man das Wasser, welches davon dufgelbst enthült, mit Hoblensaurem Kalk sättigt, wodurch man sulfoglycerinsauren lalls erbält.

Mon Kann die Sulfonuargarin - und Sulfooleinsiiure durch zwei- bis dreimaliges $\mathbf{W}$ aschen ron cinem Ueherschurs an 
Schwefelsäure befreien, indem diese in Wasser sonst sehr löslichen Säuren bei Gegenwart von Schwefclsäure unlüslich sind. In reinem Wasser aufgelöst besitzen diese beiden Sëuren einen anfangs fetten, dann stark bitteren Geschmack. In Altohol sind sie ebentalls löslich. Mit Hali bilden sie löslicbe, mit Natron, Ammonials und anderen Basen unlösliche Sulze.

Die Trennung dieser beiden Säuren ist mir unmöglich gewesen, und ich denke man wird die Ursache dieser Unmöglichkeit leicht einsehen, wenn man erst weifs, dafs diese Körpẹr schon unter den schwächsten Einflüssen sioh zersetzen. Ich werde nichtsdestowenigher im Verlaufo dieser Abhandlung die Eigenschaften und die Zusàmmensetzung einer der beiden Säuren gebon, deren Bildung mir gelang, indem ich eine Fettsäure geradezu mit Schwcfelsâure behandelte. Die in dièsean Falle entstehende Säure ist mit-der durch Bohandlang des Oels mit Schwefelsäure erhaltenen identisch.

Ich babe erwähnt, dafs die Sulfomargarin- und Sulfooleinsäure in Wasser löslich seyen. Die Wirkung dieses Lo6sungsmittels auf die Bestandtheile derselben ist jedoch der Art, dafs sie eine Zersetzung derselben herbeiführt. - Schon durch baltes Wasser werden sie nach längerer Zeit in Fettsäuren uod Scliwefelsäure zerlegt, währond kochendes Wasser dieselbe Zersutzung augenblicklich hervorbringt.

Die Fettsäuren, welche durch die Zersetzung der Sulfomargario. und Sulfoolcinsäure in der Mältc entstehen, sind jedoch nicht dieselben, welche bei der Zersetzung dieser Siiuren in der Hitze crzeugt werden.

Ich verbueite mich zunächst über die hei der Zersetzang in der Kailtc entstehenden Säuren, welche ich Motamargarin. sijure und Metaulcinsäıre genannt habe. 


\section{Metamargarinsäure.}

Ucberlälst man eine Auflüsung der Sulfomargarin - und Sulfonleinsiuure sich selbst, so bemerkt man nach einigen Stunden eino Trübung, indem ein Gemische von Metamargarinund Metaoleinsäure einen Bodensate bildet, welcher sich immer vermehrt, so dafs nach einigen Tagen die Zersetzung beinahe rollständig ist.

Die Metamergarinsäure wird ron der Metaoleinsäure mit Hülfe der Presse auf das Leichteste getrennt. Diese Reinigung ist um so leichter ausufübren, als die Metaoleinsöure nur wenig Metamargarinsäure aufgelöst hält. Eine vollkommene Reinigung der Margarineäure erreicht man durch wiederholtes Unturystallisiren dorselben in Althohol. Ans der Auflösung in Letztcrem erbält man sie in schönen, vollhommen weifsen Krystallen, welcho zweiertei Gestalt onnehmen. Zuweilen suh ich sie in glänzendea Schuppen sich nieder. schlogen, während man jedoch in den meisten Fällen dieselben eu rundlichen Warzen vereinigt erhält, ein Unterschied der Irystellbildung, der lediglich darch die Concentration der Auflösung bedingt ist.

Die so erhaltene Melamargarinsäure ist unlöslich in Wasser, löslich in Weingeist und Aetber. Ihr Schmelzpunlt ist bei $50^{\circ}$. Zur Bestimnung meiner Schmelzpunkte nebme ich den Augonblich, wo der Körper aur dem flüssigen in den festen Zustand ubergeht, und init Hinweisung auf Che rreul, der dasselbe Verfabren angenommen hat, enthalie ich mich aller weitern Auseinandersetzungen der Yortheile, welche es vor Andorn darbietet.

Lüßst man die Metamargarinsäure Jangsam erkalten, so lurystallisirt sie in glänzenden, verworrenen Nadeln.

Ich fand für diese Säure folgende Zusammensetzung: 
1.

II. III.

$\begin{array}{llll}\text { Verwenilete Säure }=0,203 & 0,214 & 0,237 \\ \text { Wasser - . . }=0,235 & 0,244 & 0,271 \\ \text { Kohlensäure . . }=0,552 & 0,50 & 0,645\end{array}$

woraus man in 100 Theilen findet:

\begin{tabular}{ccccc}
1. & II. & III. & Atome. & Theorie. \\
$\mathbf{C}=75,00$ & 74,906 & 75,2 & 70 & 75,109 \\
$\mathrm{H}=12,69$ & 12,650 & 12,6 & 140 & 12,262 \\
$\mathbf{O}=13,31$ & 12,444 & 12,2 & 9 & 12,629 \\
\hline 100,00 & 100,000 & 100,0 & & $100,000$.
\end{tabular}

Man sieht hieraus, dafs diese krystallisirten Säuren ein Atnm Wasser meht als die Margarinsäure enthalten. In den Salzen besitzt diese Săare jedoch mit der Margarinsäure genau dieselbe Zusammensetzung.

Ich hobe ron denselben das Bleisalz und das Silbersalz untersucht:

I.

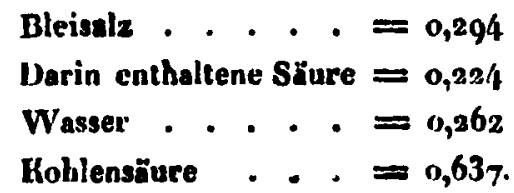

II.

III.

Silberalz $=0,36$,

Säure . . $=0,25 \dot{8}$

Wasser $\cdot=0,310$

Kohlensüure $=0,733$.
Silbersalz $=0,37 !$

Säure . . $=0,190$

Wasser $=0,225$

Kohlensäure $=0,534$.

Aus diesen Analysen leitet man folgende Zusammensetzung ab:
I.
II.
III.
Atome.
Theorie.

$$
\begin{array}{lrr}
\mathbf{C}=78,5 & 78,6 & 77,8 \\
\mathbf{H}=13,3 & 12,9 & 13,1 \\
\mathbf{O}=\mathbf{8 , 2} & 8,5 & 9,1 \\
\hline 100,0 & 100,0 & 100,0
\end{array}
$$$$
\begin{array}{rr}
70 & 78,38 \\
134 & 12,59 \\
6 & 9,03 \\
\hline 100,00
\end{array}
$$ 
Die Atomgewichtbestimmungen dieser Säuren wurden ans den Analysen der Blei-, Silber-, Kalk- und Baryt-Salze genommen. Ich mafs jedoch gestehen, dafs alle Sättigungscapacitäten, welche ich genommen babe, die Annahme des Atomgewichtes dieser Säure $=6787$ oder gleich dem doppelten A tomgewicht der Margarinsäure bestimmten. In den metamargarinsauren Kali- und Natronsalzen habe ich immer diese Menge von Shüre auf ein Atom der Basis gefunden.

Metamargarinsaures Kali. Mon erhält dieses Salz bei dem freiwilligen Verdunsten einer Iö̈sung desselben in ALkohol in kleinen, harten Hörnern, welcbe mit dem margarinsauren Ifali teine Aehnlichkeit haben. Die Zusammensetzung desselben wird Jurch einen grolsen Ueberschufs ron Kali in der geistigen lösung nicht verändert, sondern mạn findet immer 6787 , verbunden mit cinem Atom Basis.

lch fïge hier die Analyse dieses Salzes an:

$$
\begin{aligned}
& \text { Salz . . . . }=0,525 \\
& \text { Schwefelsaures hali }=0,078 \\
& \text { Hali . . . . }=0,043 \\
& \text { Säure . . . }=0,483 \\
& \text { Atomgewicht . . }=6783 \text {. }
\end{aligned}
$$

Das neutuale metamargarinsaure Kali ist in haltem Wasser wenig, in kochendem Wasser ziemlich leicht löslich. Es bchmilzt noch ebo es zersetzt wird.

Metamargarinsaures Natron und Ammoniak hrystallisiren etwa auf dieselbe Weise wie das Kalisalz. Die übrigen Salze dieser Säure sind unlöslich und bieten nichts Eigenthümliches dar. Metamargarinsaurer Kalk and Baryt fallen zuweilen mit Wasser rerbunden aieder.

\section{Metaolcinsïure.}

Die Metaoleinsäure wird duch freiwillige Zersetzung der Sultooleinsäure erhalten, und man trennt sie leicht von der 
Margarincänre, welche sie eathalten bann, indem man sie einer Temperatur von $-2^{\circ}$ his $-3^{\circ}$ aussetzt. Die Reinigung Ajeser Säure ist defshalb um so leichter, als sie bei gewơhn. licher 'Temperatur nur äufserst wenig Metamargarinsänre aufGelüst erhalten kann.

Sie ist gewöhnlich flüssig, gelblich gefärbt, unltslich in Wesser, löslich in Aether, in Alkohol aber aur sehr werrig Jislich.

Die letztere Eigenschaft ist in der Art ron Wichtigheit, als sie dient die Metaolainsiure von der Oleinshiure zu unterscheiden, welche sich bekanntlich in Alkobol in allen Verhältnissen 16st. Der Destillation unterworfen zersetzt sie sich unter Bildung von Producten, mn welchen ich im Verlaufe dieset $\Lambda$ hhandlung noch sprechen werde.

Die Metaoleinsăure besitzt folgende Zusammensetzmng :

$$
\begin{aligned}
& \text { Säure }=0,209 \\
& \text { Wasser }=0,225 \\
& \text { Kohlensëure }=0,573 .
\end{aligned}
$$

In 100 Theilen:

Atome. Theorie.

$$
\begin{array}{rrr}
C=75,8 & 70 & 75,9 \\
\mathrm{H}=11,9 & 128 & 11,3 \\
\mathrm{O}=12,3 & 9 & \frac{12,8}{100,0}
\end{array}
$$

woraus man sicht, daßs diese Säure zwei Atome Wasser mehr als das Hydrat der Oleinsäure enthält, welches durch $\mathrm{C}_{90} \mathrm{H}_{i_{26}} \mathrm{O}_{8}$ ausgedriickt wird.

Ich habe die Säure auch in Verbindung mit Basen analysirt und führe bièr die Analyse des metaoleinsauren Silberoxydes an:

$$
\begin{aligned}
& \text { Salz } \cdot=0,283 \\
& \text { Staurc } \cdot=0,223 \\
& \text { Wasser } \cdot=0,349 \\
& \text { Hohlensïure }=0,623 .
\end{aligned}
$$


In 100 Theilen:

$$
\begin{aligned}
& \mathbf{C}=77,3 \\
& \mathbf{H}=13,2 \\
& \mathbf{O}=10,6 \\
& \hline 100,0
\end{aligned}
$$

Atamc. Thearie.

\begin{tabular}{rr}
70 & 77,3 \\
126 & 11,3 \\
8 & 11,4 \\
\hline & 100,0
\end{tabular}

Nimmt man dernnach 6936,2 als Atomgewicht dieser Sïure an, so vorliert sie, indem sie sich mit Basen rerbindet, ein Atom Wasser.

Diese Zahl ist übrigens anch dieselbe, wolohe ioh ous den Sättigungscapacitäten whalton habe.

Man wird einsehen. mit welchen Schwierigkeiten ich bei der Bestimmung des Atomgewichts zu hämpfen hatte, da er mir durchaus unmöglich war, eic brystallisirtes metaoleinsares Salz zu exhalten. Dis Salze des Hali, Natrons und A mmoniaks sind die einzigen löalichen Salze, und zoigen keine Neigung zur Krystallisation.

Iacbdem ich biermit die beiden Fottsäuren, welche sich durch Zersetzung dor Sulfomargarin- und Sulfooleinsäure in der Halte erzengen, beschrieben bobe, komme ich auf moine frühore Bemerkang zurüch, dafs nämlich die Zersetzung ganz anderer Art ist, vena die Sulfomargarin - und Sullonleinsäuro viner Temploratur von $100^{\circ}$ ansgesetzt verden.

In diesem Falle findet eine augenblichlicht Zersetzong in a neve Fottuäuran Statt, weiche ich, wegen ihres gröfsorn Wassergehaltes als die vorbergehenden, Hydranargurin- und Hydroclainsture genonnt habe

Ihre Darstellung ist sebr einfach. Wean man die AufJösungen der Sulfomargarin - und Sulfooleineäure Hochen lafat, 80 sind sie in wenigen Minuten vollstïndig zersetzt. Dio beiden Fettaluren werden sodann unter die Presse gebracht, um die Hydromargarinsäure rollkommen frei von Hydrooleinsäure zu erhalten, za welchem Zwecke ich nitunter bei ciner Temperatur von $20^{\circ}$ bis $30^{\circ}$ prefste. Zur vollstündigen Reinigung 
dicser Säure ist jedoch ein mehrmoliges Umkirgstallisiren der. selben in Alkohol erforderlich, indem die Hpdrooloinsiure bei weitem löslicher in demeelben iat, als die Bydromargarinesure.

\section{Hydromargarinsäure.}

Die Hydromargarinsäure, welche man anf diese Weise erhält, ist woifs, unlöslich in Wasser, löelich in Altohol und Aether, and hrystallisirt daraus in dicken, länglichen Warzen. Ihr Schmelzpunkt ist boi $60^{\circ}$ gefunden wowden. Läfat man sie nach dem Schmeizen erkalten, so gesteht sie einer un. durchsichtigen, aber Krystallinischen Manse, wolche mit der Beschmolzenen Metamargarinsïure nicht ver'wechsell werden hann, da diese beinahe durohsichlig, eratere aber vollhommen undurchsichtig ist.

Ihre Zusammensetzung ist wie folgt:

\section{II. II}

$\begin{array}{lll}\text { Säure } \cdot \cdot=0,164 & 0,248 & 0,254 \\ \text { Wasser } \cdot=0,297 & 0,285 & 0,283 \\ \text { KohLensäure }=0,705 & 0,663 & 0,675\end{array}$

Demuach in 100:

I. II. III. Atome. Theorie.

\begin{tabular}{lllrr}
$\mathbf{C}=73,82$ & 73,91 & 73,49 & 70 & 73,93 \\
$\mathrm{H}=12,46$ & 12,75 & 12,36 & 142 & 12,24 \\
$\mathbf{O}=13,72$ & 13,34 & 14,15 & 10 & 13,83 \\
\hline 100,00 & 100,00 & 100,00 & & 100,00
\end{tabular}

Mit Besen verbunden gibt diese siure oin Atom Wacers $a b$, wie aus der Aualyse der Salke hervorgeht, welche ich hier anführen will:

I.

Hydromargarinsaure Bleioxyd $=0,290$

Sïure darin . . . . . $=0,222$

Wakser . . . . . . . $=0,257$

Hohlenoüure . . . . . $=0,605$. 
II.

Hyaromargs, Silber $=0,294$

Sirwe darin . . $=0,24$ !

Wasser . . . $=0,278$

Kohlensäure . . $=0,655$.
III.

Hydromargs. Silber $=0,290$

Säure darin . . $=0,212$

Wesser . . . $=0,237$

Koblensäure . $.=0,575$.

In 200 Theilen entsprechen diesen Analysen:
1.
II.
III.
Atome.

$$
\begin{array}{lllr}
\mathbf{C}=75,2 & 75,14 & 74,98 & 70 \\
\mathbf{1 1}=12,8 & 12,80 & 12,40 & 140 \\
0=12,0 & 12,06 & 12,63 & 9
\end{array}
$$

Das Atomgewricht dieser Säure habe ich aus dem gilber -, Bhi- und dem lfalksalze bestimmt, und dieselben Rücksichten, deren ich bei der Metamargarinsäure erwähot habe, hestimmten mich das Atomgewicht dieser Säuse auf 7123 festzusetzen.

Alle krystallisirten hydromargarınsauren Salze sind aus einem Atom Basis und einer Säuremenge gebildte, welcheq durch 7122 vorgestellt wird. Man erhält diese Zahl aus den Anslysen des hydromargarinsauren Kali, Natron und Ammonialss, welche lrystallisirt sind.

Bei der Destillation zersetzt sich die Hydromargarinsïure, und geht wieder in Metamargarinsäure über, und ich führe defshalb die Analyse einer Säure an, welche durch Destillation aus volltommen reiner Hydromargarinsäure erhalten wurde, die dabei gänzlich in Metamargarinsäure äberging, während zugleich cine augenfällige Entwichclung ron Wasser stattfand.

In 100 :
Säure $\cdot=0,260$
C $=75,23$
Wasser . $=0,300$
$\mathrm{H}=12,60$
Kohlensüure $=0,731$.
$0=12,17$.

Das hydromargarinsaure Kali krystallisirt aus Alkoho! in derben Warzen.

Bei all den verschiedenen Proben diescs Sulzes, woiche 
ich der.Analyse unterworfen habe, fand ich immer, dals mit einem Atom dor Basis sich 7122 Gewichtstheile Säure verbunden hatten. . Das Salz bot dieselbe Zusammensetzung dar, auch wenn es aus einer vässerigen, sebr alkaliscben Lösung krystallisirt war. Das hydromargarinsaure Hali kann ohne Veränderung gcschmolzen werden. In Wasser ist es 2 war wenig löslich, doch wird durch einen Ueberschuf ron Alkali seine Löslichkeit sehr vermehrt.

Hydromargarinsaares Natron und Ammonials krystallisiren ebenfalls mit Leichtigheit aus Alkohol, in Gestalt ron hleinen, Lrystallinischen Körnern. Alle übrigen bydromargarinsauren Salze sind in Wasser gar nicht, und in Alkohol nar sohr wenig löalich.

\section{Hydrooleinsiture.}

Wie sohon frühes erwähnt wande, ist diese Strole ein Zersetzungsprudust der Solfooleinaïure, bei einer Temperatur von $100^{\circ}$. Fs genügt, dieselbo längere Zeit einer kültenan Temperatur auszusctzon, um sie von noch etwas anbängenuer Hydromargarinsäuc bel'reit zu arhalten. Die aufgelöate $\mathbf{H y - ~}$ dromargarinciure hetrigt übrigens sohr wenig, denn bei einer Bebandlung des Olivenöls, 20 welcher drei Pfund desselben rerwendet wurden, gelang ea mir, zwei Pfund Hydromargariusäure und folglich etwa cin Pfund Iydrooleinsäure zu erhalten.

Die Hydradainsäure bietet in reinem Zustande folgende Eigenschaften day s sie ist etwas gefärbt, und besitzt immer einen äthcrartigen Geruch; in Wasser ist sie unlöslich, wäh. rend sie vom Alkolsol in jedem Verhältnils aufgenommep wird, wodurch sie allein schon von der Metaoleinsiivare hiareichend unterschieden ist.

Ibre Zusammensetzung ist: 
I.

$$
\begin{array}{ll}
\text { Säure } \cdot=0,246 & 0,942 \\
\text { Wasser } \cdot=0,262 & 0,260 \\
\text { Hohlensäure }=0,658 & 0,651
\end{array}
$$

entsprecbend in 100 Theilen ausgedrüclt:

$$
\begin{array}{rrrr}
C=73,9 & 74,38 & \text { Atome. } & \text { Theorie } \\
H=11,7 & 11,92 & 130 & 74,47 \\
O=14,6 & 13,70 & 10 & 13,4.1 \\
\hline 100,0 & 100,00 & & 100,00 .
\end{array}
$$

Mit den Oxyden des Bleies und silbers verbunden, verliert sio Wasser and gibt:

I.

Bilbersaiz $=0,929$

Sirure . . $=0,175$

Waster . $=0,197$

Hohlendiure $=0,470$
11

Bleisaix . $=0,245$

Sture . . $=0,2,0$

Wasser . $=0,238$

Mohlensilure $=0,504$

Woraus men fur 100 findet:

$$
\begin{aligned}
& \text { I. } \mathrm{n} \text {. Atome. Theorite. } \\
& \begin{array}{llrr}
6=-75,5 & 75,33 & 70 & 75,9 \\
H=12,1 & 11,86 & 198 & 11,3 \\
O=12,1 & 12,81 & 9 & 12,8 \\
\hline 100,0 & 100,00 & & 100,0 .
\end{array}
\end{aligned}
$$

Wan wird hier leicht elnen Unserschied zwischen dom erhaltenen und den aus der Theorie abgeleitcten Zablen finden. Diels findet seine Ursache in der grolsen Sehwierigheit, welche die Analyse dieser Salzo darbletet. Mon erhult sie allo in einem syrupartigen Zoulande, was mre Austrocknong and beanders ihre Verbresnnung ausnehmend schwlerig macht. Dr Atomgewicht habe ich $=7028$ gefunden.

Die hydrooleinsauren Salzo haben mit denen der Meta. oleinsüure sebr viele Analogie, obgleich die hydrooleinsauren 
Kali-, Natrbn und Ammoniak-Salze in Wasser bei weilèm löslicher sind, als die entspriechenden metaoleinsauren Salze

Wird die Hyerooleinstare der Destillatlon unterworfen, so wird sic aul dieselbe Weise nie die Metaoleinsïure zersetzt, und was von det Einen gesagt wird, iut durchgehends auch auf dic Andere anwendbar.

Wenn dro Destillation dex Hydröoleinsäure vorsichtig an. gestellt wird, indem man für die Verdichtung der Producte Sorge trägt, so fizdet eine ziemtieh regelmäfsig̨e Enlwickelung ron reiner Hohlensäure Statt, und in der Vorlage sielıb man eine lileine Menge yon Wasser sich ansammeln, auf welcher eine ölighte Schicht bemerkbar ist. Diese Schicht wird vornehmlich von zwe hüssignen Kohlenwasserstoffen gebildet, die mit dem ölbildenden Gase gleiche elementare Zusammensetzung habel. aber auf andere Weise verdichtet sind, Die eine Verbindung kocht bei $55^{\circ}$, die Andere bei 110

Ich hobe Erstere Olsin, die Letztère Elain genannt. Diese Namen wahlte ich in der Veherzeugung. dals sich diese Pro. ducte in allen Fällen bilden, wo Oel oder Oleinsäure destil. lirt werden. Ich beschreibe hier den Weg, welchen ich eingcsclilagen habe, um ihre Trennung zu bewerkstelligen.

Voresst begann irh mit der Destillation der ölariigen Schleht hivi ener Temperatur yon 150 wodurch das Olein und Elain von einem empyreumatischen Oele befreit werden, welches sich immer bildet. Hierauf wurden sie mit einer ziemfich verdïnnten Auflösung ron Kali geschültelt, üm einige Spuren fliichtiger Feltsäuren zu entternen, womil sie begleitet sind, und sodann das Ganze mit Chlorcalcium digeriet. Nachdem sie so von allen Treurden Stoffen befreft waren, blieb mir, um beide von einander zu trennen, thein anderer $W$ eg, als die Verschiedenhcil ihrer Hochpunkte zu benutzen, und es ist nicht zu sagen wieviel wiederholter Destillationen es bedurfte, um diese Körper rein zu erhaiten. Man glaubt nicht 
wie schwierig die Trenaung dieser beiden flüchtigen Staffe ith, obgleich sio mit oinem Tnterschiede ron $50^{\circ}$ Lochen. Nichtsdestoweniger wurden sie endlich rollkommen rein und mit beständigem Kochpunkte erhalten. Bevor sie zur Analyse rerwendet wurden, brachte ich inner etwas Kalium hinzu, da ich fand, dafs man auf diese Weise eine Destillation über dieses Metall ersparen hann, welche zur gänzlichen Reinigung dorselben erforderlich ist.

\section{Olein.}

Des Olein ist vollkommen weifs, Aüssig, leichter als $W$ asser, sehr dünnflüssig, von durchdringendem, arsenikartigem, nouseösom Geruch. Es ist sebr entzündlich und verbreant mit einer Flamme, die zuweilen ins Grünlicho spielt. In Wesser ist es hanm, in Alkohol and Aether sehr leicht löslich. 8chwefelsäure scheint heine Einwirkung auf dasselbe anazuübor. Sein Hocbpunkt ist bei $55^{\circ}$. Auf die Lebensverrichtungen scheint es eines nachtheiligen Einflufs auszuüben, denn Vögel, welche einige Zeit dessen Dämpfe ein. atbmeten, fielen todt hin.

Bei der Analyse gab es folgende Zahlen:

\begin{tabular}{|c|c|c|}
\hline \multirow[t]{2}{*}{$\begin{array}{l}\text { Olein } \cdot=0,708 \\
\text { Wawer } \cdot=0,276 \\
\text { Hohlensiare }=0,645\end{array}$} & $\begin{array}{c}\text { In soo: } \\
\mathbf{C}=\mathbf{8 5 , 7 2} \\
\mathbf{H}=\mathbf{1 4 , 7 ^ { 3 }}\end{array}$ & \multirow[t]{2}{*}{$\begin{array}{l}\text { Atome. } \\
\text { C } \\
\mathrm{H}_{2}\end{array}$} \\
\hline & & \\
\hline
\end{tabular}

Die Bestimmung der Dichtigkeit seinea Dampfes gab folgondes Resoltat:

$$
\begin{aligned}
& \text { Kugel mit Luft . . }=46,540 \\
& \text { Kugel mit Dampf . . }=46,809 \\
& \text { Inhalt der Kugel . . }=180,8 \mathrm{C} \text {. C. } \\
& \text { Zurückgeblicbene Luft . }=3, \text { C.C. } \\
& \text { Darometer . . . . }=0,771 \mathrm{M} \text {. } \\
& \text { Thermometer . . . }=14,5 \text {. }
\end{aligned}
$$


Berechnet man noch die Zahlen, so findet man die Dichtigbeit gleich 2,875 . Niment man an, dals das Volumen durch $\mathrm{C}_{3} \mathrm{H}_{6}$ dargestellt wird, so fünde man $2,9 / 2$. Bei andern Dichtigkeitsnahmen habe ich mitunter 3,1 2 und 3,05 erhalten, was einer schwer zu entferneriden Spur von Elain zugeschrieben werden mufs.

Des Olein verbindet sich in der Hälle mit Chlor und bildet cin flüssiges Chlorür von älherartigem Geruch, welches ich jedoch erst wenizr untersucht habe, da ich einer hinlänglichen Menge reinen Oleins entbehrte. Die Schwefelsäure scheint indessen, selhst bei der Kochhitze, das Olein unangetastet $\mathrm{zu}$ lassen.

\section{Elain.}

Dieser lörper ist weifs, unlöslich in Wasser, löslich in Aether, in Alkohol weniger löslich als das Olein, von durch. dringendem Geruch, der sich ron dem des Oleins nur wenig unterscheidet. Das Elain brennt mit einer schönen weifsen Flamme; es kocht bei $110^{\circ}$. Es ist leichter als Wasser, und wird, wie es scheint, von Schwefelsüure nicht angegrillen.

Analyse des Elains:

Atome. Theoric.

$$
\begin{array}{lll}
\mathbf{C}=85,40 & \mathrm{C} & 85,95 \\
\mathrm{H}=14,66 & \mathrm{H}_{2} & \frac{14,05}{100,04}
\end{array}
$$

Bei der Dichtiglieitsbestimmung seines Dampfes erhielt.ich:

Kugel mit Luft . . . $=45,756$

Kugel mit Dampf $\cdot=46,261$

Inhalt der Kugel..$=196$, C. C.

Barometer . . . $\cdot=0,77^{2} \mathrm{M}$.

Thermometer. . . $=16$,

Dichtigkeit . . . . $=4,488$, welche cinem

Annal. d. Pbarin. XX. Bds. 2. Heft. 
Volumen gleich $\mathrm{C}^{3} \mathrm{H}^{10}$ entopricht, und berechnet 4,904 geben mufote. Han wird zugs:ben, dafs es sehr schwierig ist, eine genauere Dichtigkeitsnabme zu erreichen, dean trotz aller Vorsicht bei der oben erwähnten Scheidang des Elains ron Spuren des darin enthaltenen Oleins, dürfte dicse doch mitunter unmöglich seyn.

Nit dem Chlor verbindet sich des Elain zu cinem flüssigen Chlorür.

Ich habe dieses Chlurür erhalten, indem ich das Elain einem Strom des Gases aussetzte, wobei eine grofse Wärmeentwickelung stattfand. Ich brachte zu dissem Endzweche Elain in eine tubulirte Retorte und destillirte dasselbe in einem Chlorgasstrom. Das von anhängender Salzsäure befreite Elainchlorü: ist schwerer als Wasser, und scheint sich nicht in demselben aufzulösfn. Is besitzt einen ätherartigen Geruch, und brennt mit intensir grüner Flamme. Es wurde aus gìichen Volumen Chlor und Elain bestehend gefunden,

Die Analyae gab Folgendes:

$$
\begin{aligned}
& \text { Elainchlorïr }=0,309 \\
& W \text { asser }=0,259 \\
& \text { Koblensäure }=0,632 .
\end{aligned}
$$

Chlorbestimnung :

$$
\begin{aligned}
& \text { Elaincblorür }=0,383 \\
& \text { Chlorsilbir }=0,519 \\
& \text { Chlor . }=0,197 .
\end{aligned}
$$

Zusammensorzung in 100 Theilen:

$$
\text { Atome. T'heorie. }
$$

$$
\begin{aligned}
& \text { c. . }=56,5 \\
& \text { H. . }=9,3 \\
& \text { C } 5 \\
& 57,4 \\
& \text { Cblos }=33,3 \\
& \text { II } 10 \\
& 9,4 \\
& \text { cl } \\
& \frac{33,2}{100,0 .}
\end{aligned}
$$

Die's wären aisu die Producte der Destillation der Motaslein- und Hydrooleinsäure. I's würde schr zu wïssohen 
seyn, der Zusemmensetrang dieser beiden Kohlenwasserstoffe anch des Wasser und dioghKohlensäure hinzufïgen zu können, doren Bildung ich za gleicher Zeit uachgewiesen habe, und durch eine Formel die Zerfallung jener Bärper' in diese Producto sa versinnlichen. Es ist aber gewifs, dafs dergleichen Fermela nur dann von Werth sind, wenn sie sich auf gonaue Angaben der Mengen der gebildeten Producte stützen, welches mir in dem rorliegenden Falle unmöglich gewesen ist.

Wenn, wie Alles uns nnnehmen läfst, die Fetteïuren bel einer hŏhern Temperatur zersetzt, die Bildung solcher Kohleswasserstoffgase veranlassen, die sich bei der Zersetzung der Oelo in der Bothglühhitze, erzeugen, so scheint es mir nicht uninteressant, aus ein und demselben Körper isomerische Kohlenwasserstoffe entstehen $\mathrm{zu}$ sehen, dic jedoch bei den rerschiedenen Temperaturen, welchen sie ihre Fitstehung rerdankien, rerschiedene Verdichtung erleiden.

Es bleibt mir ondlich nur noch dic Autzählung einiger Versuche über die Verbindung der Schwefelsäure mit den Fettsäuren zu erwähnen übrig, und da die Untersuchung der durch Zersetzung dieser Säuren gebildeten ISörper der Zweclı dieser Abhandlong ist, werde ich dis Analyse eines derselben anführen.

Ich habe indessen gefunden, dals alle Fettsäuren die Eigenschaft besitzen, sich mit der Sehwefelsäure zu verbinden, und bin defshalb der Meinung, dafs das Studium dieser Verbindung der Gegenstand einer besondern Untersuchung zu werden verdient, welcher ich mich nöchstens zu unterziohen gedentue.

Die flüssigen Fettsüuren verbinden sich am leichtestell, und zwar in der lläite, nit der Schwefelsäure, und die neugebildeten Säuren bestehen dann gewőhnlich ans einem Atom Fettsäure und At. Schwefelsäure. Ich fand ferner, dals diese Säuren gleiche Eigenschəften mit jenon besitzen, welche 
sich bilden, wenn geradezu Oel mit Schwefelsïure behandelt wird, und es ist sehr wahrscheinlich, dafs dic, entweder aus Jem Oele, oder aus den blofsen Fettsüıren dargestellten Säuren nur durch verschiedencn Wassergehalt sich unterscheiden.

Ich gehe zur näheren Beschreibung einer solchen Süure, die darch Behandeln der Metaoleinsäure mit Schwefeltäure erhalten wurde, über.

Die Verbindung findet unter Wärmeentwickelung Slatt, so dafs man das Gefäls, in welchem sie vorgeht, mit eincr Kältemischung umgeben mufs. Dic hier gebildete Sulfooleinsäure hann selbst wiederholt mit Wasser gewaschen werden, da ein Ueberschufs an Schwefelsäure ih火e Auflösung in Wasser verhindert.

Zuletzt wird die Säure durch das Wasser zersetzt, und wenn diese Zersetzung in der Kältc vor sich geht, so ist das Product Metaoleinsäure, während sich bei Anwendung von Wärme Hydrooleinsäure bildet. Die Auflösung dieser Säure besitzt einen sehr bitteren Geschmack, und scheint keine Irrystalle zu liefern. Ich habe sie unit Basen verbunden analysirt, and führe hier die Analyse des Barytsalzes on:

$$
\begin{aligned}
& \text { Sulfooleinsaurer Baryt }=0,306 \\
& \text { enthält Säure . . . }=0,251 \\
& \text { giht Wasser . . . }=0,264 \\
& \text { Kohlensäure . . . }=0,656 \text {. }
\end{aligned}
$$

Bestimmung der Schwefelsäure :

$$
\begin{aligned}
& \text { Salz . . . . . }=0,697 \\
& \text { schwefelsaurer Baryt }=0,102 \\
& \text { Schwefelsäure } \cdot \cdot=0,035
\end{aligned}
$$

woraus man fïr 100 die Zusammensetzung findet:

$$
\begin{aligned}
\mathbf{C} & =7^{2,2} \\
\mathbf{H} & =11,6 \\
\mathbf{O} & =10,2 \\
\text { SO }_{3} & =6,1
\end{aligned}
$$


was folgender Formel entspricht:

$$
\mathrm{C}_{70} \mathrm{H}_{226} \mathrm{O}_{2}+\mathrm{SO}_{3}
$$

und dieser Theorie nach würde die Zusammensetzung in 100 Theilen seyn:

$$
\begin{aligned}
\mathbf{C} & =71,9 \\
\mathbf{H} & =10,5 \\
\mathbf{O} & =10,9 \\
\text { SO }_{8} & =6,7 .
\end{aligned}
$$

Man sieht leicht, dafs diese Zusammensetzung durch die Formel $\mathrm{C}_{3 s} \mathrm{H}_{120} \mathrm{O}_{3}+3\left(\mathrm{H}_{2} \mathrm{O}\right)+\mathrm{SO}_{2}$. ausgedrücht werden kann, nämlich durch Oleinsäure, Wasser uml Schwefelsäure. Bei der ausgedehnteren Untersuchung, welche ich auf die Verbindungen der Fettsäuren mit der Schwefelsäure zu ver. wenden gedenke, werde ich von der freiwilligen Zersetzung der Sulfooleinsäure mit Alkohol snrechen, da dieselbe, nach cirigen vorläufigen Versuchen, merkwürdig zu seyn seheint,

\section{Untersuchung der Amniosflüssigkeit eines dreimonatlichen Pferdefötus; von C. Vogt in Bern.}

Din Stute, wovon die Flüssigkeit genommen war, litt an Iühmung des Bückenmarks und der hinteren Extremitäten. Sie wurde def'shalb getödtet, die Geschlechtstheile herauspräparirt und die Amniostlüssigkeit vorsichtig durch ein nöbrchen abgezapft. Aus Unvorsichtigkeit des Dieners war die Allantoisflüssigleit laider verloren gegangen.

Ich erhielt die Flüssiglicit etwa zwei Stunden nach dem Tode des Thieres durch die Güte des Herrn Professor Gerher. - Sie hat einen nur sehr schwachen, thierischen Geruch, eine gelbliche Farbe, war aber gaoz hell und klar. Thr 